\title{
The efficiency of varying parameters when optimizing reinforced concrete structures
}

\author{
Ashot Tamrazyan, and Anatoly Alekseytsev* \\ Moscow State University of Civil Engineering, Yaroslavskoe shosse, 26, Moscow, 129337, Russia
}

\begin{abstract}
The actual issue is considered related to the purpose of the type of variable parameters, their number and grouping of structural elements in the optimization of reinforced concrete structures. The genetic search algorithm is used to solve the problem of minimizing the cost of frame systems. The rate of convergence of the iterative procedure is taken as one of the criteria for the significance of the parameter when varying. The comparison of optimization results for a reinforced concrete beam and a frame is given. A conclusion is made about the expediency of varying parameters of different types and their ranking is performed from the standpoint of the goal function sensitivity to parameter changing.
\end{abstract}

\section{Introduction}

One of the effective methods to find rational solutions for reinforced concrete structures are algorithms [1-5]. They allow to think of variable parameters as discrete sets of values. In addition, for real civil structures, the number of variable parameters necessary to obtain a rational solution can be very large. Various tools are under development to address these issues in research methods. These are, for example, the use of penalty functions $[6,7]$, strategies for filtering construction variants with irrational values of the target criterion [8], the technology of adaptive self-grouping members [9], the use of regulated genetic operators [10-12], etc. All of these approaches, to one degree or another, overcome the problem of deterioration of convergence for particular optimization problems. However, the question of the application of a specific method of research algorithms for the optimization of reinforced concrete structures seems difficult due to the large calculating capacity of the problems to be solved. There is a need to develop some simplified estimation method that can predict which parameters need to be changed in a given problem, how many of those parameters should be in order for us to get the greatest effect of optimization while ensuring an acceptable amount calculation. Moreover, for the problems of large computing capacity, it is important to choose these types and the number of parameters for which it is possible to obtain a rational solution. This problem, despite the growth in computing capacities of computers, still prevents the use of genetic algorithms and other optimization methods to solve the problems of finding rational solutions for reinforced concrete structures.

In this article, is proposed a ranking of the parameters according to their contribution to the improvement of the objective function, which will make it possible to advance in the

*Corresponding author: aalexw@mail.ru 
solving of the optimization problem for high calculation capacity tasks. A calculation scheme from [13] is used as the basic algorithm. When seeking a solution, genetic mutation operators $[14,15]$ without the use of adaptive properties, the crossover operator [16] with a cut point and the selection operator based on the roulette wheel [17-20] are used. To keep the best solutions, the principles of elitism are used [21, 22].

\section{Optimization problem solution method}

When optimizing a frame structures, one of the important tasks is to determine the types of variable parameters that most significantly affect reducing the cost of the structure when finding a solution. When optimizing systems with a large number of variable parameters, the question of choosing the types of parameters is decisive for the possibility of obtaining a solution. Let's consider the following types of settings:

- concrete class;

- reinforcement steel grade;

- dimensions of the structural element cross-section;

- the area of the working rebars section.

The number of variable parameters is also influenced by the number of unifying groups of structural elements; this number does not change in the process of finding a solution. Reinforced concrete structures shown in Fig. 1.
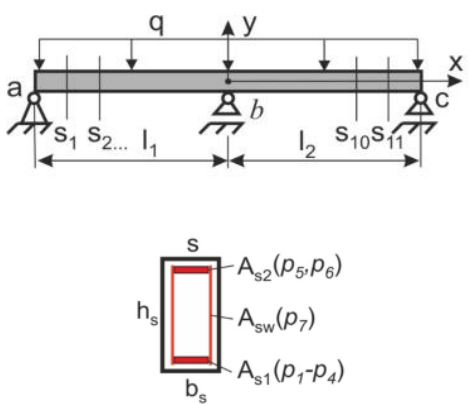

a)

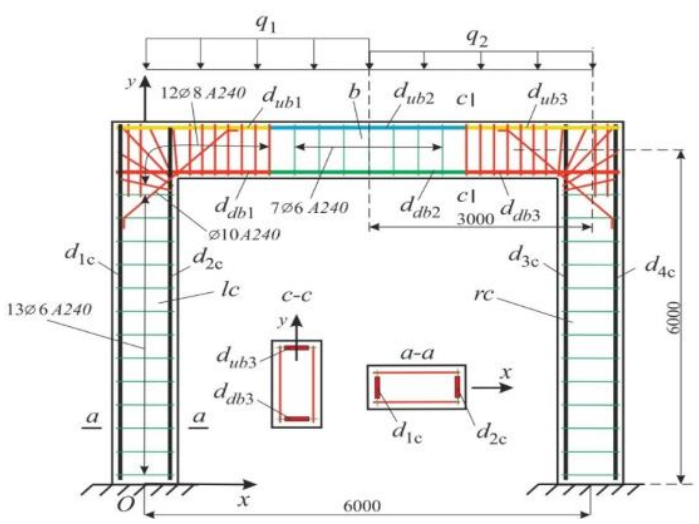

b)

Fig. 1. Structures under study: two-span beam (a), planar frame (b)

As the criterion that determines that the solution will not improve during the iterative process, we take the number of iterations in which there have been no changes in the base of the best design structures:

$$
N=\sqrt[n]{3} \sqrt[3 n !]{ }
$$

where $m$ is the average from discrete sets the number of the variable parameter values, $n-$ is the number of variable parameter.

\section{Optimal designing results}

Let us consider changes in the cost of a structure using examples of optimization of a beam system with discrete sets containing four classes of concrete, 4 grades of reinforcement, 4 or 7 groups of unification and 5 possible combinations of section sizes with reinforcement schemes. In figures 2, 3 the designations are introduced: I - is a the number of iterations 
required to obtain a rational solution; $C_{I}$ - is a cost of the best design option at the current iteration of the genetic algorithm; $\Delta C=C_{I-1}-C_{I}-$ is the difference between the costs at the previous and current iterations.

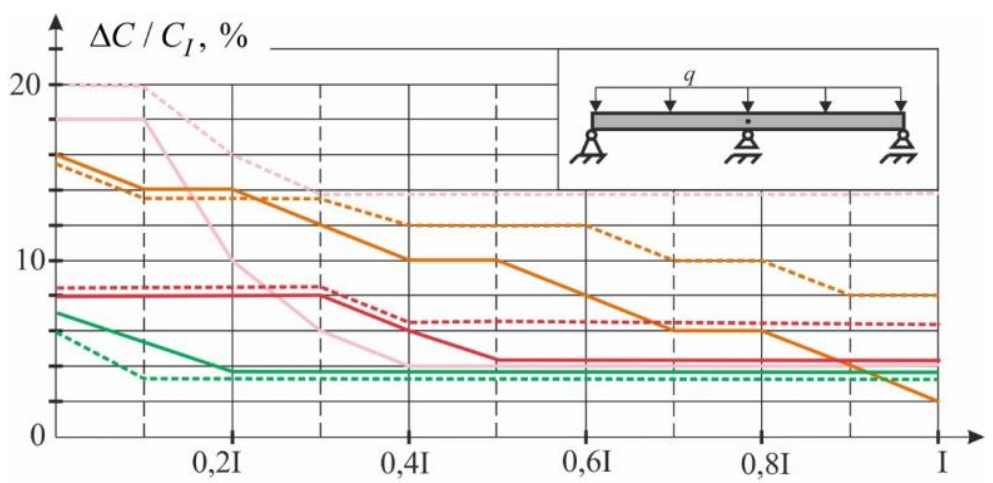

Fig. 2. Influence of the variable parameter type on the relative decrease in the cost of the beam (designation of colors and types of lines is shown in Fig. 3)

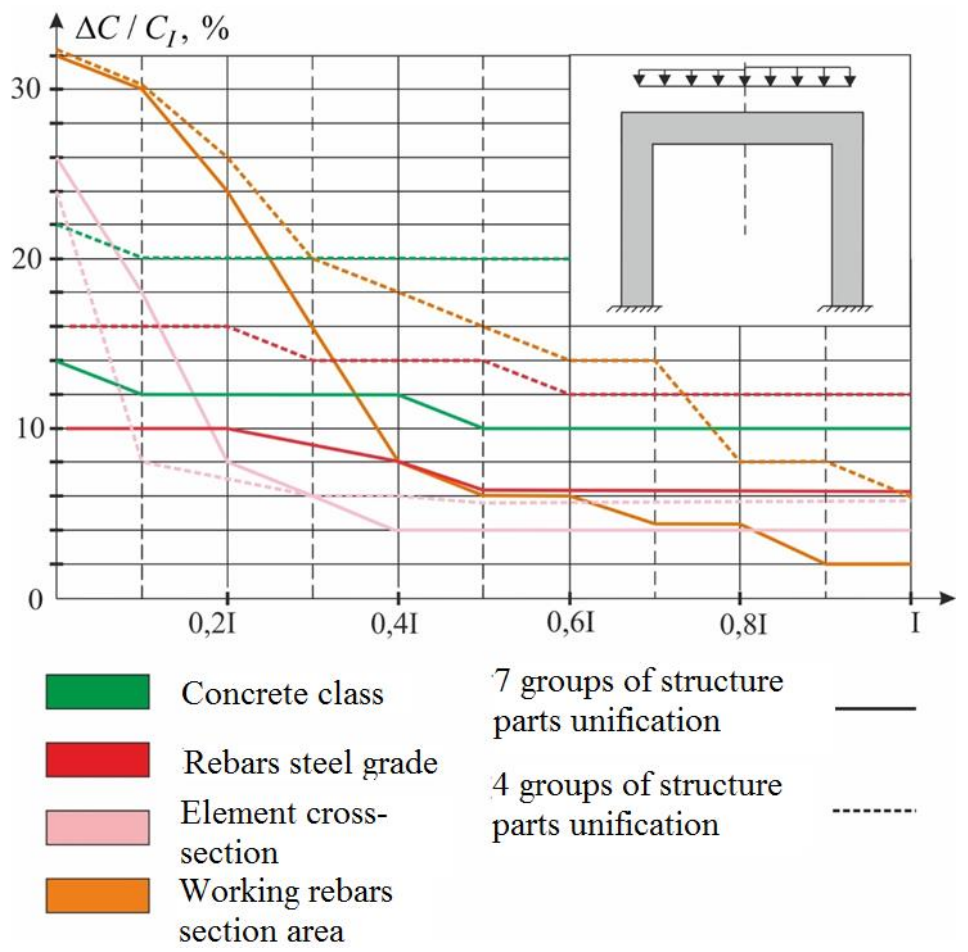

Fig. 3. Influence of the variable parameter type on the relative reduction in the cost of the frame

Horizontal sections of lines on the charts mean that the cost of the best option has not changed, but the set of the best solutions has changed. The slopes in the graphs are a linear approximation of the stepwise changes in cost value. Let us explain the results obtained. Each graph illustrates the effect on the cost of a variable parameter with the other parameters unchanged. 
When varying the class of concrete, the cost changed by about $3 \%$, while changes occurred at the initial stage of research, an increase in unification groups did not lead to significant changes in the results. The change in reinforcement grades during optimization had the same qualitative effect on the evolution of the cost, which was $4 \%$.

At the same time, an increase in unification groups, as well as for one class of concrete, led to a proportional decrease in cost. The variation in the dimensions of the structural elements cross-section has a significant effect on the modification of the value of the goal function. At the same time, for four unification groups it was $6 \%$, and for 7 groups it was already $14 \%$. In this case, a decrease in value was observed of $30 \%$ of the total number of iterations. The cross section of the working reinforcement has the greatest impact on the cost. So, with 4 groups of unification of structural elements, the cost has changed by $8 \%$, and with 7 - by $14 \%$. This decrease was observed practically over the entire search for a solution. Taking into account the basis, qualitatively similar results were obtained.

Depending on the degree of the various parameters influence on the changes of the objective function, it is possible to classify these parameters according to their importance (Table 1).

Table 1. Ranking of variable parameters

\begin{tabular}{|l|c|c|c|c|c|}
\hline \multicolumn{1}{|c|}{$\begin{array}{c}\text { Type of the } \\
\text { parameter }\end{array}$} & \multicolumn{2}{|c|}{$\Delta C / C_{I}, \%$} & \multicolumn{2}{c|}{$\tilde{I} / I, \% *$} & $\begin{array}{c}\text { Target rank } \\
\text { (higher is better) }\end{array}$ \\
\hline & beam & frame & beam & frame & \\
\hline Concrete class & 3 & 4 & 20 & 50 & 1 \\
\hline Rebars steel grade & 4 & 6 & 20 & 50 & 3 \\
\hline $\begin{array}{l}\text { Dimensions of } \\
\text { cross-section }\end{array}$ & 14 & 22 & 30 & 50 & 4 \\
\hline $\begin{array}{l}\text { the area of the } \\
\text { working rebars } \\
\text { section }\end{array}$ & 14 & 28 & 90 & 100 & \\
$*$
\end{tabular}
\begin{tabular}{l}
$\tilde{I}-$ the number of iterations up to which there was an improvement in the goal criterion \\
\hline
\end{tabular}

The question under consideration is no less important when solving multicriteria optimization problems. For each of the target criteria, it is necessary to determine the influence of the type of variable parameters on compliance with the constraints for a variant of a constructive solution. The degree of this influence can be used to predict the value of the constraint violation penalty for a given optimization criterion. In fig. 4. for a reinforced concrete beam, the influence of restrictions on strength, crack opening width and stiffness is taken into account. Obviously, the greatest impact on stress violation is exerted by varying the structural element section sizes and section areas of the working rebars.

\section{Discussion}

Until now, when solving optimization problems based on genetic algorithms, the ranking of parameters by their influence on the change of the objective function was not taken into account. This could lead to a significant increase in the quantity of calculations due to the insufficiently fast convergence of the iterative process. For problems of significant calculation capacity, which correspond to real structural systems of reinforced concrete buildings, it is difficult and sometimes even impossible to obtain optimal solutions without taking into account the rank of the variable parameters. Therefore, it is advisable to develop the classification methodology proposed for other building structures of metal, stone and other materials. The issues of studying the effectiveness of varying and ranking parameters for optimization taking into account special influences $[23,24]$ remain unexplored. 


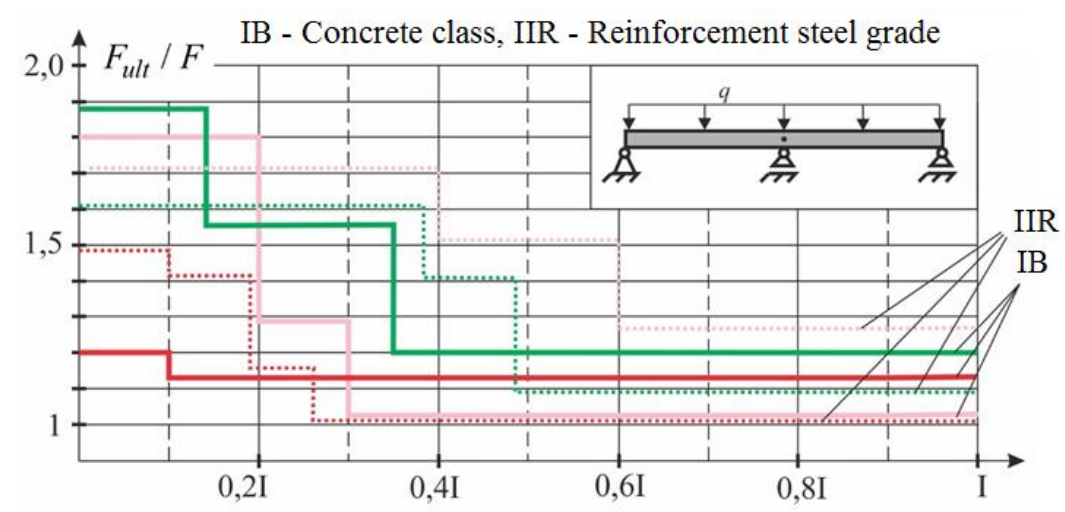

III- Working rebars section area

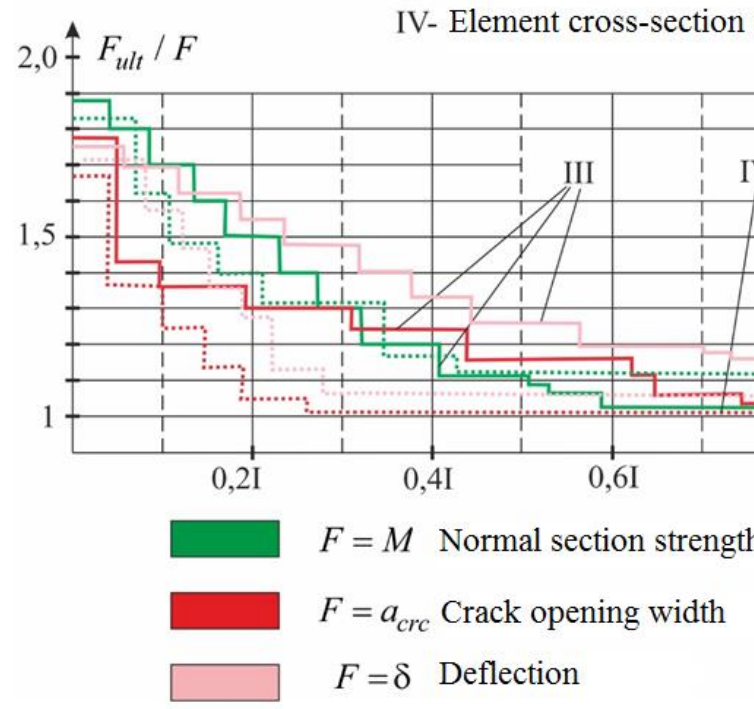

Fig. 4. Influence of variable parameters on the limit constraints margin

\section{Conclusion}

Studies have shown that the most important from the point of view on cost reduction effectiveness is the variation in the dimensions of the sections and work rebars areas. The results illustrate that the variation of the concrete class significantly and sharply affects the deflection and strength of the structure, therefore, their change can lead to an improvement of the design solution only at the initial stages of research and with a small number of classes. For problems with a large number of variable parameters, for a faster approach to the region of feasible solutions, it is advisable not to introduce these parameters as variables. The effect on the limitations of the rebars area variation and the dimensions of the section is smoother; therefore, it is advisable to introduce a greater number of values of the variable parameters to improve the stability of the convergence and to overcome the local extrema.

The authors express their gratitude to the organizing committee of the conference and reviewers for the opportunity to publish the research results. 


\section{References}

1. Mangal, M., Cheng, J.C.P. Automation in Construction. doi:10.1016/j.autcon.2018.01.01302380-x (2018)

2. Zhang, G., Ali, Z.H., Aldlemy, M.S., Mussa, M.H., Salih, S.Q., Hameed, M.M., AlKhafaji, Z.S., Yaseen, Z.M. Engineering with Computers. doi:10.1007/s00366-02001137-1 (2020).

3. Kirsanov, M., Serdjuks, D., Buka-Vaivade, K. Lecture Notes in Civil Engineering 70, p. 25-35 (2020)

4. Alekseytsev, A.V., Al Ali, M. Magazine of Civil Engineering, 2018, 83(7), стр. 175185

5. Tamrazyan, A., Alekseytsev, A. E3S Web of Conferences, 2019, 97, 04005

6. Zinkova, V.A. Lecture Notes in Civil Engineering, 2021, 95, стр. 213-218

7. Alekseytsev, A., Botagovsky, M., Kurchenko, N. E3S Web of Conferences, 2019, 97, 03002

8. Tamrazyan, A., Alekseytsev, A. IOP Conference Series: Materials Science and Engineering, 2020, 869(5), 052027

9. V. Togan*, A.T. Daloglu Computers and Structures 86 (2008) 1204-1218

10. Prokurov, M., Indykin, A., Alekseytsev, A. MATEC Web of Conferences, 2018, 251, 04017

11. Lushnikova, V.Y., Tamrazyan, A.G. Magazine of Civil Engineering, 2018, 80(4), стр. $128-137$

12. Gibb, S., La, H.M., Louis, S. in (2018) 2018 IEEE Congr. Evol. Comput. CEC 2018 Proc., . doi:10.1109/CEC.2018.84777909445(2003) 129:1(105)

13. A.V. Alekseytsev, S.A. Akhremenko, Magazine of Civil Engineering 5 (81), 32-42 (2018)

14. Loganina, V., Fediuk, R., Vatin, N. $2020 \quad$ IOP Conference Series: Materials Science and Engineering 896(1), 012001

15. Tamrazyan, A., Alekseytsev, A. IOP Conference Series: Materials Science and Engineering, 2020, 869(5), 052019

16. Klyuev, S.V., Shlychkov, D.I., Muravyov, K.A., Ksenofontova, T.K. 2020 International Journal of Advanced Science and Technology 29(5), c. 2577-2583

17. Serpik, I., Alekseytsev, A. IOP Conference Series: Materials Science and Engineering, 2018, 365(5), 052003

18. Kirsanov, M.N. $2020 \quad$ Magazine of Civil Engineering 96(4), c. 110-117

19. Fan, Z., Xia, L., Lai, W., Xia, Q., Shi, T. 2019. Structural and Multidisciplinary Optimization. 59 (2), pp. 647-658. doi:10.1007/s00158-018-2090-4

20. Tamrazyan, A.G. IOP Conference Series: Materials Science and Engineering, 2018, 365(5), 052021

21. Assimi, H., Jamali, A., Nariman-zadeh, N. 2017. Swarm and Evolutionary Computation. 37 pp. 90-103. doi:10.1016/j.swevo.2017.05.009

22. Camp, C. V., Pezeshk, S., Hansson, H. Flexural 2003. Journal of Structural Engineering. doi:10.1061/(asce) 0733

23. Tamrazyan, A.G., Avetisyan, L.A. IOP Conference Series: Materials Science and Engineering, 2018, 365(5), 052034

24. Tamrazyan, A., Avetisyan, L. Applied Mechanics and Materials, 2014, 638-640, p. 6265 